\title{
Die Zukünfte von Gender Mainstreaming
}

\author{
GERTRAUDE KRELL
}

Wenn es um die Frage nach der Zukunft von Gender Mainstreaming geht, sollten wir unterscheiden zwischen Gender Mainstreaming als einem gleichstellungspolitischem Konzept und Gender Mainstreaming als einem Prinzip oder Gebot. Mit diesem Beitrag möchte ich das erläutern und zugleich die Zukünfte beider Varianten erörtern. ${ }^{1}$

Gender Mainstreaming ist sowohl ein Prinzip als auch ein Konzept zur Neu-Gestaltung des Geschlechterverhältnisses. ${ }^{2}$ Dabei steht der Begriff „Gender“ dafür, dass dieses Verhältnis nicht als naturgegeben, sondern als historisch-gesellschaftlich hervorgebracht betrachtet und behandelt wird. Gender Mainstreaming als Prinzip und Konzept zielt darauf, Entscheidungs- und andere Prozesse so zu gestalten und zu bewerten, dass die Beteiligten dabei den Blickwinkel der Gleichstellung der Geschlechter einnehmen. Diese Definition stammt vom Europarat. Von diesem und der Europäischen Kommission wurde seit Mitte der 1990er Jahre die Verbreitung des - ursprünglich aus der Entwicklungspolitik stammenden Konzepts Gender Mainstreaming vorangetrieben: Im Sinne einer „Doppelstrategie“ soll Gleichstellung als Querschnitts- oder Gemeinschaftsaufgabe die bisherige Frauen- und Gleichstellungspolitik ergänzen (nicht ersetzen!), um dadurch besser in den „Hauptstrom“ vordringen und diesen verändern zu können.

Meine These lautet, dass sich trotz der skizzierten Gemeinsamkeiten von Gender Mainstreaming als Prinzip und als Konzept die Zukünfte beider Varianten unterscheiden.

\section{Die gegenwärtige Situation in Deutschland}

Mit Blick auf Deutschland ist zunächst festzuhalten, dass in privatwirtschaftlichen Unternehmen Gender Mainstreaming als Konzept kaum realisiert ist. Wo dort überhaupt Gleichstellungspolitik existierte, wurde in der Regel „Frauenförderung" durch "Chancengleichheit" oder auch "Chancengleichheit und Diversity“ und schließlich „Diversity“ ersetzt. Gender Mainstreaming fand und findet sich vor allem in öffentlichen
Verwaltungen und Hochschulen - aber auch in Organisationen wie Gewerkschaften.

Diejenigen, die Gender Mainstreaming als Konzept propagieren und praktizieren, sind mit drei (miteinander zusammenhängenden) Herausforderungen konfrontiert: Im Recht (zum Beispiel im Allgemeinen Gleichbehandlungsgesetz - AGG) werden neben Geschlecht auch andere soziale Kategorien berücksichtigt, die diskriminierungsrelevant sind. Nicht nur aufgrund ihres Geschlechts, sondern auch aufgrund anderer Kategorisierungen haben sich benachteiligte Gruppen zu sozialen Bewegungen zusammengeschlossen, aus denen ebenfalls spezielle Gleichstellungspolitiken hervorgegangen sind und die auch zu rechtlichen Regelungen geführt haben. Und schließlich ist Gender mit diesen anderen Kategorien verschränkt.

\section{Rechtliche Regelungen}

Eine Herausforderung besteht darin, dass Gender Mainstreaming als alleiniges gleichstellungspolitisches Konzept nicht dem sogenannten „horizontalen Ansatz“ im Recht entspricht, der mehrere Kategorien gleichberechtigt nebeneinanderstellt. ${ }^{3}$

Um nur zwei Beispiele zu nennen: Laut $\$ 67$ Abs. 1 BPersVG (Bundespersonalvertretungsgesetz) ist die Interessenvertretung verpflichtet, daran mitzuwirken, dass ,jede Benachteiligung von Personen aus Gründen ihrer Rasse oder wegen ihrer ethnischen Herkunft, ihrer Abstammung oder sonstigen Herkunft, ihrer Nationalität, ihrer Religion oder Weltanschauung, ihrer Behinderung, ihres Alters, ihrer politischen oder gewerkschaftlichen Betätigung oder Einstellung oder wegen ihres Geschlechts oder ihrer sexuellen Identität unterbleibt“. In $\$ 1$ AGG ist als dessen Ziel festgeschrieben, „Benachteiligungen aus Gründen der Rasse oder wegen der ethnischen Herkunft, des Geschlechts, der Religion oder Weltanschauung, einer Behinderung, des Alters oder der sexuellen Identität zu verhindern oder zu beseitigen“. Insofern wäre eine zwar geschlechtsneutrale Stellenanzeige, in der aber „Deutsch als Muttersprache“ oder eine bestimmte Altersgruppe als Anforderungen benannt werden, nicht AGG-konform.

\section{Weitere gleichstellungspolitische Konzepte}

Die Existenz weiterer gleichstellungspolitischer Konzepte, die sich jenseits des Geschlechts auf andere ggf. diskriminierungsrelevante Merkmale beziehen, ist ebenfalls eine Herausforderung für Gender Mainstreaming. Auch für (Inter-) Cultural Mainstreaming oder auch Interkulturelle Öffnung, Sexual Mainstreaming, Age Mainstreaming, Disability Mainstreaming gibt es in vielen Organisationen inzwischen Programme, Maßnahmen und Zuständigkeiten. Hinzu kommen Diversity Mainstreaming oder auch anders bezeichnete Diversity-Konzepte. Wie Gender Mainstreaming sind diese Konzepte ebenfalls aus politischen Bewegungen hervorgegangen. Und das gilt, entgegen einem weit verbreiteten Vorurteil, auch für Diversity.

\section{Verschränkungen von Gender mit anderen ungleichheitsrelevanten Kategorien}

Vor einigen Jahren äußerte der Leiter einer Behörde bei einer Podiumsdiskussion zu Diversity, er habe einen Zielkonflikt, weil er einerseits mehr Frauen und andererseits auch mehr Migranten befördern solle. Das „Dann nehmen Sie doch Migrantinnen“ kam aus dem Publikum.

1 Für ihre sehr hilfreichen Überarbeitungsvorschläge danke ich Christina Klenner und Gudrun Linne.

2 Diese kurze Einführung basiert auf: Krell, G./ Mückenberger, U./Tondorf, K. (2011): Gender Mainstreaming: Chancengleichheit nicht nur für Politik und Verwaltungen, in: Krell, G./Ortlieb, R./Sieben, B. (Hrsg.): Chancengleichheit durch Personalpolitik, 6. Aufl., Wiesbaden, S. 85- 104.

3 Im Grundgesetz wird Geschlecht zwar privilegiert, aber auch dort werden weitere Kategorien berücksichtigt. 
Die mit diesem Beispiel angesprochenen Verschränkungen von Gender mit anderen ungleichheitsrelevanten Kategorien werden in der Frauen und Geschlechterforschung unter Etiketten wie „Intersectionality“, eingedeutscht „Intersektionalität“, oder auch „Interdependenz“, behandelt.

„Intersektionalität“ kommt, wie Diversity, aus den USA. Wurzeln des Ansatzes sind die Kritik zum einem am Anti-Diskriminierungsrecht, das der (Mehrfach-)Benachteiligung schwarzer Frauen nicht Rechnung trägt, und zum anderen am (Mainstream-)Feminismus, weil er durch weiße Mittelschicht-Frauen dominiert wird. ${ }^{4}$ Deshalb stehen im Zentrum von „Intersectionality“ die drei sozialen Kategorien „race, class, gender“ und deren Zusammenwirken, wenn es um Benachteiligungen oder auch Privilegierungen geht. Im deutschen Sprachraum geht es im Kontext „Intersektionalität“ um die Fragen nach dem „Wie“ dieses Zusammenwirkens und nach weiteren ungleichheitsrelevanten Kategorien wie ethnische Zugehörigkeit/Migrationshintergrund oder Behinderung. Nicht zuletzt wird darüber gestritten, ob auf die Fragen nach der Auswahl und Gewichtung (oder auch Hierarchisierung) solcher Kategorien überhaupt eine immer und überall gültige Antwort gegeben werden kann - oder sollte. $^{5}$

Anknüpfend an diese Diskussionen über Intersektionalität betrachtet eine Gruppe von deutschen Forscherinnen „Gender als interdepen-

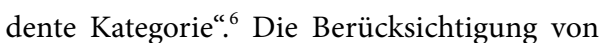
Verschränkungen soll noch radikaler erfolgen, indem für Ungleichheiten nicht Verhältnisse $z w i$ schen Kategorien bedeutsam gemacht werden, sondern innerhalb einer Kategorie. Weitere Kategorisierungen sollen also gleichsam in das Inne-
4 Vgl. z. B. Crenshaw, K.W. (2010 [1989]): Die Intersektion von "Rasse" und Geschlecht demarginalisieren: Eine Schwarze feministische Kritik am Antidiskriminierungsrecht, der feministischen Theorie und der antirassistischen Politik, in: Lutz, H./Herrera Vivar, M. Th./Supik, L. (Hrsg.): Fokus Intersektionalität, Wiesbaden, S. 33-54.

5 Vgl. z. B. Walgenbach, K. (2007): Gender als interdependente Kategorie, in: Walgenbach, K./ Dietze, G./Hornscheidt, A./Palm, K.: Gender als interdependente Kategorie: Neue Perspektiven auf Intersektionalität, Diversität und Heterogenität, Opladen/Farmington Hills, S. 23-64; Klinger, C./Knapp, G. A. (2008): ÜberKreuzungen, Münster; Winker, G./Degele, N. (2009): Intersektionalität, Bielefeld; Smykalla, S./Vinz, D. (Hrsg.) (2011): Intersektionalität zwischen Gender und Diversity, Münster. re von Gender - sowie von allen anderen Kategorien - hineinverlagert werden. Mit Blick auf Gender folgt daraus, dass es keine „reine“ Frauenund Geschlechterpolitik (oder auch -forschung) geben kann, sondern dabei immer auch weitere Unterscheidungen sowie daran geknüpfte Bewegungen und Politiken berücksichtigt werden müssen. Mit Blick auf alle anderen Kategorien oder Gruppierungen gilt entsprechend, dass innerhalb dieser das Geschlecht einen Unterschied macht, dem in Forschung und Praxis angemessen Rechnung zu tragen ist - ganz im Sinne von Gender Mainstreaming als Prinzip.

\section{Ausblick: Hat Gender Mainstreaming ausgedient?}

Das ist ganz sicher nicht der Fall. Durch die Berücksichtigung weiterer Kategorien, sei es fein säuberlich von Gender getrennt, mit Gender verschränkt oder innerhalb der Kategorie Gender gewinnt demnach Gender Mainstreaming als Prinzip an Bedeutung. Die Zukunft von Gender Mainstreaming als gleichstellungspolitischem Konzept hängt davon ab, wie - im Einzelfall - auf die geschilderten Herausforderungen reagiert wird. Grundsätzlich bestehen hier folgende Möglichkeiten:

(1) Unter dem Dach Gender Mainstreaming werden auch andere Kategorien berücksichtigt - und damit Gender als interdependente Kategorie be trachtet und behandelt. Das scheint mir aber nur dort eine realistische Perspektive zu sein, wo noch keine weiteren gleichstellungspolitischen Konzepte und Zuständigkeiten existieren. Denn wo es diese gibt, sind die Vertreterinnen und Vertreter

dieser Konzepte genauso von deren Notwendigkeit und Existenzberechtigung überzeugt wie die $\mathrm{Ak}$ teurinnen und Akteure des Gender Mainstreaming von diesem Konzept.

(2) Die zweite Möglichkeit wäre: Konzepte wie Gender Mainstreaming, Interkulturelle Öffnung usw. bleiben weiterhin nebeneinander bestehen Bei dieser Möglichkeit gibt es noch einmal ein großes Spektrum möglicher Varianten: von einem gänzlich unkoordinierten Auftreten und Handeln bis hin zur Kooperation im Rahmen eines Netzwerkes, Runden Tisches oder Ähnlichem.

(3) Ein dritter möglicher Politikansatz besteht darin, Gender und andere Kategorien - und damit alle Konzepte oder Politiken - unter dem Dach Diversity zu integrieren. Hier gibt es neben gleichstellungspolitisch Engagierten, die gegen Diversity argumentieren und agieren, ${ }^{7}$ auch Frauen- und Gleichstellungsbeauftragte, die die Einführung und Ausgestaltung von Diversity-Konzepten selbst in die Hand nehmen. ${ }^{8}$

Bei Variante drei hat Gender Mainstreaming als eigenständiges gleichstellungspolitisches Konzept keine Zukunft. Aber Gender Mainstreaming als Prinzip bleibt weiterhin notwendig, um zu gewährleisten, dass Gender nicht nur als eine (Kern-)Dimension von Diversity betrachtet und behandelt wird, sondern auch bei allen anderen Dimensionen von Diversity der Blickwinkel der Gleichstellung der Geschlechter eingenommen wird. Das gilt gleichermaßen für Variante zwei. Denn Ältere, Menschen mit Migrationshintergrund, Menschen mit Behinderung und andere Gruppen bestehen weder nur aus Männern noch aus geschlechtslosen Wesen. Insofern geht es auch mit Blick auf Gleichstellungspolitiken für diese Gruppen immer um die Frage, ob Ungleichheiten der Geschlechter vernachlässigt, verfestigt oder verkleinert werden. Und genau dafür steht Gender Mainstreaming als ein Prinzip mit Zukunft.

7 Für eine kritische Auseinandersetzung mit diesen Argumenten: Krell, G. (2010): Gender unter dem Dach Diversity: Eine Auseinandersetzung mit häufig geäußerten Einwänden, in: HohmannDennhardt, Ch./Körner, M./Zimmer, R. (Hrsg.): Geschlechtergerechtigkeit. Festschrift für Heide Pfarr, Baden-Baden, S. 147-157; vgl. auch Krell, G. (2014): Gender und Diversity: Eine Diskursgeschichte, in: Funder, M. (Hrsg.): Gender cage revisited: Handbuch zur Organisations- und Geschlechterforschung, Baden-Baden, S. 319-342.

8 Vgl. z. B. Bender S. F./Wolde, A. (2013): Diversity Policies: Implementation mit Brüchen, in: Bender, S. F./Schmidbaur, M./Wolde, A. (Hrsg.): Diversity entdecken. Reichweiten und Grenzen von Diversity Policies an Hochschulen, Weinheim/Basel, S. 126-144. 